\title{
32-km distributed temperature sensor based on Brillouin loss in an optical fiber
}

\author{
X. Bao, D. J. Webb, and D. A. Jackson \\ Applied Optics Group, Physics Laboratory, The University, Canterbury, Kent CT2 7NR, UK
}

Received May 3, 1993

\begin{abstract}
We present a novel distributed temperature sensor that uses the temperature dependence of the frequency at which the loss is maximized in the interaction between a cw laser and a pulsed laser. With a 32-km sensing length, a temperature resolution of $1^{\circ} \mathrm{C}$ has been achieved; it is also shown that a spatial resolution of $5 \mathrm{~m}$ may be obtained.
\end{abstract}

Among the hierarchy of sensors, the distributed fiberoptic temperature sensor is unique, as it offers continuous sensing over tens of kilometers with good temperature accuracy and high spatial resolution. Distributed temperature sensors (DTS's) that use Raman scattering as the sensing mechanism have already been reported..$^{1-4}$

More recently, the variation with temperature of the Stokes frequency shift in Brillouin scattering has been proposed as a suitable mechanism for a DTS. ${ }^{5}$ A system based on this technique has been demonstrated by Kurashima et al. ${ }^{6}$ Those authors reported a temperature resolution of $3^{\circ} \mathrm{C}$ combined with a spatial resolution of $100 \mathrm{~m}$ over $1.2 \mathrm{~km}$ of fiber for this technique, which they have termed Brillouin opticalfiber time-domain analysis (BOTDA). Higher spatial resolution and temperature accuracy combined with a significant increase in the sensing range have recently been reported ${ }^{6,7}$ with BOTDA. Our research ${ }^{7}$ has indicated that the maximum range when BOTDA is used at $1.3 \mu \mathrm{m}$ (without optical amplifiers) is restricted to $\sim 20-25 \mathrm{~km}$ because of system noise (combined electronic and optical) and the input $\mathrm{cw}$ beam power limit, which is governed by the pulsed power depletion that occurs through the Brillouin interaction. Although at $22 \mathrm{~km}$ the sensing length of the DTS described in Ref. 7 is the largest yet reported to our knowledge, there are several applications for which a longer sensing length combined with higher spatial resolution is desirable. To this end we have explored the possibility of using Brillouin loss rather than Brillouin gain. We have found that significant improvements in the performance of DTS's based on Brillouin scattering are possible with this approach. In this Letter we describe a DTS system based on Brillouin loss that has demonstrated a temperature resolution of $1^{\circ} \mathrm{C}$ with a spatial resolution of $5 \mathrm{~m}$ and a sensing length of more than $32 \mathrm{~km}$.

The system operates as follows: light from a tunable-frequency $\mathrm{cw}$ laser at a frequency $\nu_{L 1}$ (the $L$ in the subscript represents the Brillouin loss process) is launched into one end of the sensing fiber. The output from a pulsed laser at frequency $\nu_{L 2}$ is injected into the other end of the sensing fiber. When $\nu_{L 1}=\nu_{L 2}+\nu_{B}$ ( $\nu_{B}$ is Brillouin frequency), the counterpropagating beams interact through the
Brillouin gain mechanism. ${ }^{8}$ The pulsed beam is amplified at the expense of the cw beam; hence the intensity of the cw beam will be reduced as a result of the Brillouin interaction.

If the intensity of the $\mathrm{cw}$ beam emerging from the fiber is monitored following the launch of the pulsed beam, a decrease in the intensity will be observed whenever Brillouin loss occurs. The time delays between the launch of the pulsed beam and those regions where power is transferred from the $\mathrm{cw}$ beam correspond to round-trip times for light traveling to and from the regions of Brillouin loss. These times provide the positional information. If the laser frequency difference is adjusted, then the cw light will experience loss in parts of the fiber at a different temperature. Hence by slowly scanning one of the laser frequencies it is possible to map out the temperature distribution of the whole fiber.

In our previous system ${ }^{7}$ the $\mathrm{cw}$ laser frequency was lower than that of the pulsed laser and therefore experienced gain. When depletion of the pulsed light can be neglected, the two approaches are equivalent for sensing purposes, giving signals that differ only in sign and not in magnitude. However, the Brillouin loss approach is superior when much of the fiber is at the same temperature, as may often be the case. In such a situation, when Brillouin gain is used, the pulsed beam is depleted by the interaction, resulting in a very weak signal from the end of the fiber most distant from the pulsed source. Conversely, with Brillouin loss the pulsed beam is increased as a result of the interaction, resulting in a much stronger signal from this end of the fiber and thus permitting the realization of longer sensing lengths.

The experimental arrangement is illustrated in Fig. 1. Both lasers were solid-state cw diode-pumped Nd:YAG ring lasers emitting close to $1319 \mathrm{~nm}$. The maximum launched power of the $\mathrm{cw}$ beam laser was $\sim 10 \mathrm{~mW}$. The frequency of the laser could be adjusted by temperature tuning the cavity. A Bragg cell was used to provide short optical pulses ranging in time from 50 to $400 \mathrm{~ns}$. The peak launched pulsed power from the first-order diffracted beam was $\sim 5 \mathrm{~mW}$. This signal is monitored at photodetector $\mathrm{D} 1$ and is also used to synchronize the start of the data storage. The zero-order beam was mixed with 


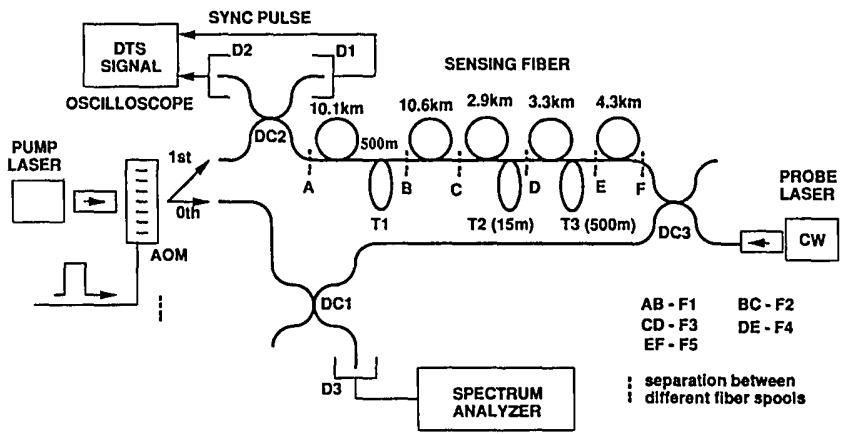

Fig. 1. Experimental arrangement: AOM, acousto-optic modulator.

light from the cw beam laser by directional coupler DC1 and detected with a fast $(20-\mathrm{GHz})$ detector. The resultant beat frequency was monitored with a microwave spectrum analyzer.

Most of the pulsed power was launched into one end of the $32.2-\mathrm{km}$ sensing fiber by a $90: 10$ coupler, DC2, while the cw beam was injected into the other end of the fiber by a variable-ratio coupler, DC3. The cw light emerging from the sensing fiber was detected with a low-noise photoreceiver with a $125-\mathrm{MHz}$ bandwidth amplifier D2 and monitored with a storage oscilloscope. Ideally the sensing fiber would be fabricated from a single length of fiber to ensure that the propagation constants, such as the mode field diameter, attenuation, and dispersion, are the same throughout the sensing length. The actual $32.2-\mathrm{km}$ fiber sensing length consisted of five different fiber spools, F1-F5 (see Fig. 1), all spliced together. The specifications of the fiber spools were different, which meant that the Brillouin frequency shifts were different even when all the fiber was at the same temperature. Most of the sensing fiber $(32.2 \mathrm{~km})$ was at ambient temperature. However, three separate sections of the sensing fiber were placed in temperaturecontrolled ovens, as shown in Fig. 1. There were nine splices over the whole fiber length. All optical launching and delaunching were achieved with $20 \times$ microscope objectives. Manually adjustable polarization controllers were used at both ends of the sensing fiber to maximize the Brillouin loss signal over the whole fiber length; their use is necessary, as the Brillouin interaction is polarization sensitive.

Figure 2(a) shows two sample traces measured with the storage oscilloscope monitoring the $\mathrm{cw}$ beam intensity, synchronized with the launch of the pulsed beam into the fiber. The pulse width is $50 \mathrm{~ns}$, corresponding to 5-m spatial resolution. The data shown by the upper trace correspond to the condition in which the Brillouin loss is maximized for fibers F1 and F2 $(21.2 \mathrm{~km})$ at ambient temperature. The sharp transition in the signal that occurs at point $\mathrm{C}$ (i.e., where fibers F2 and F3 are spliced) occurs because the propagation constants of the fibers are different. The discrete steps in the trace are caused by the digital nature of the oscilloscope. In the bottom trace of Fig. 2(a) the beat frequency was chosen to maximize the Brillouin loss at $46^{\circ} \mathrm{C}$ (first oven); the third oven temperature was $54^{\circ} \mathrm{C}$.

To compare the performance obtained by using Brillouin loss against that which used Brillouin gain, we repeated the experiment with the sensing fiber subject to the same temperature distribution. Figure 2(b) shows the best trace that we could achieve with gain; in this case the minimum pulse width that could be used was $100 \mathrm{~ns}$, as the signal was so weak. The upper and lower traces of Fig. 2(b) measured with Brillouin gain can be compared directly with the traces of Fig. 2(a) obtained with Brillouin loss. Comparing the upper traces of Figs. 2(a) and 2(b), we see that with the gain process only the signal at position $\mathrm{B}$ is observable, the signals in the $\mathrm{C}-\mathrm{F}$ region of the fiber being totally undetectable. Comparing the bottom traces of Figs. 2(a) and 2(b), where the beat frequency is set to the Brillouin shift corresponding to the temperature of the first oven $\left(46^{\circ} \mathrm{C}\right)$ at position $B$, we find that the peak for Brillouin gain is much weaker and the peak at $\mathrm{E}$ is not observable. (Note that the peak seen at $\mathrm{E}$ in the Brillouin loss spectrum is at a temperature of $54^{\circ} \mathrm{C}$ and hence is not at its maximum.) These data were taken with the sample averaging facility of the oscilloscope; typically 20 averages were necessary.

The high spatial resolution of the Brillouin loss technique cannot be appreciated from Fig. 2(a), as each sample of the storage scope averages over a distance of $50 \mathrm{~m}$. To demonstrate the higher spatial resolution, we performed experiments with fiber lengths of 15,10 , and $5 \mathrm{~m}$ placed in the oven at position $\mathrm{D}$ at $24.1 \mathrm{~km}$ (temperature $46^{\circ} \mathrm{C}$ ). Again the frequency of the $\mathrm{cw}$ beam was adjusted to maximize the Brillouin loss; the measurements were taken with pulse widths of 100 and $50 \mathrm{~ns}$. The results of these experiments are summarized in Fig. 3. Figure 3(a) shows the trace that results when the 15-m length of

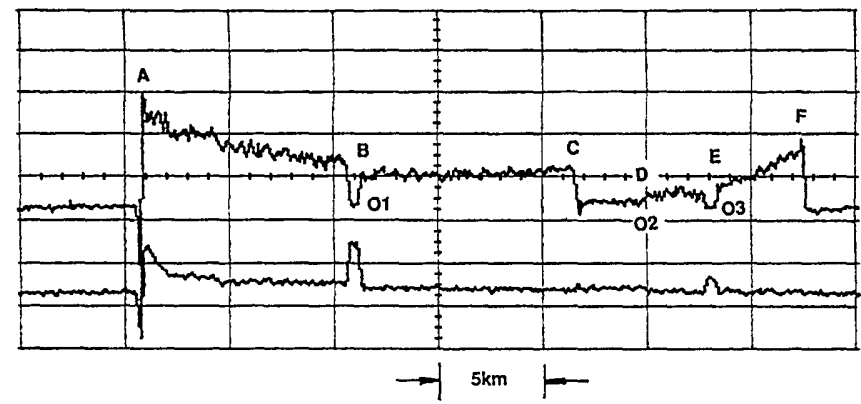

(a)

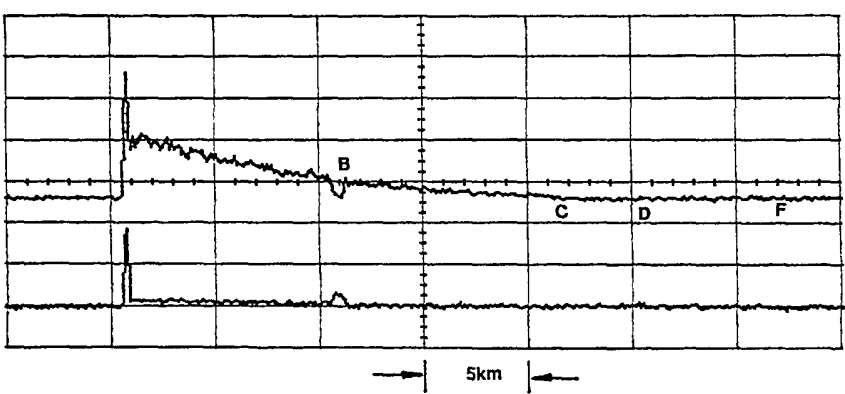

(b)

Fig. 2. Oscilloscope traces showing $\mathrm{cw}$ beam intensity monitored at D2 for (a) Brillouin loss and for (b) Brillouin gain. Time base, $50 \mu \mathrm{s} /$ division; $\mathrm{cw}$ wave frequency optimized for fiber at ambient temperature (upper traces) and at $46^{\circ} \mathrm{C}$, the temperature of the first oven (lower traces). 


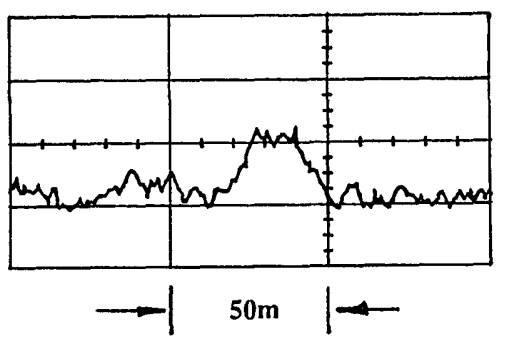

(a)

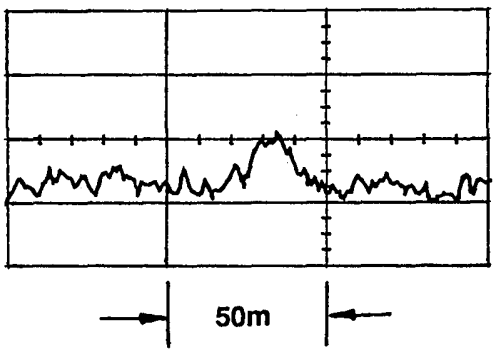

(b)

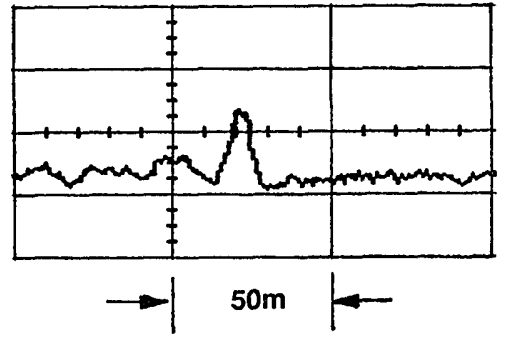

(c)

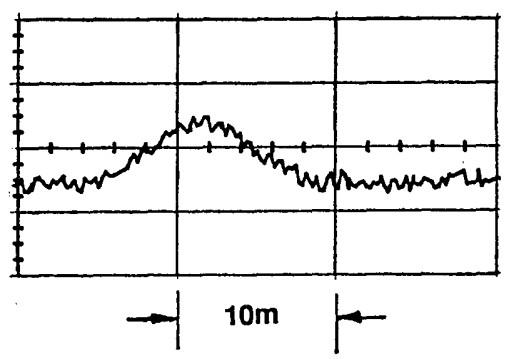

(d)

Fig. 3. Expanded view of the oscilloscope traces of $\mathrm{cw}$ beam intensity as a function of time for a section of fiber in oven 2 (fiber D) at $46^{\circ} \mathrm{C}$. One sample corresponds to $0.5 \mathrm{~m}$ [for (a)-(c)] and to $0.1 \mathrm{~m}$ [for (d)]; see text for details.

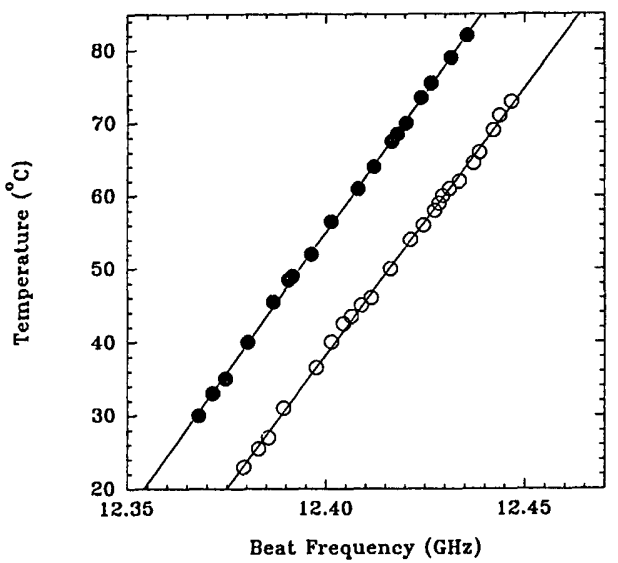

Fig. 4. Laser beat frequency that gives maximum Brillouin loss for the fibers in oven 1 (F1, filled circles) and oven 3 (F4, open circles) as a function of oven temperature.

fiber is in the oven and the pulse width is $100 \mathrm{~ns}$. Here the time axis has been expanded to provide $0.5 \mathrm{~m}$ of fiber per sample. Figure $3(\mathrm{~b})$ shows the trace with $10 \mathrm{~m}$ of fiber in the oven, again with a 100-ns pulse, and Fig. 3(c) shows the trace for the $5-\mathrm{m}$ length of fiber with 50-ns pulses; this trace is expanded further in Fig. 3(d), which provides $0.1 \mathrm{~m}$ of fiber per sample. In these traces the number of averages was increased to 512. Most workers in the field of DTS's define the spatial resolution as the rise (or fall) time of the received signal. Applying this definition to Fig. 3(d), we could claim a spatial resolution of better than $3 \mathrm{~m}$; however we prefer to define the resolution as the half-width of the minimum detectable signal, which in this case is $\sim 5 \mathrm{~m}$. The resolution is actually governed by the rise time of the Bragg cell used in these experiments. We believe that, with a faster Bragg cell, a better spatial resolution could be achieved. The fundamental spatial resolution is determined by the phonon lifetime, i.e., the reciprocal of the Brillouin half-width; this limits the spatial resolution to 1 to $2 \mathrm{~m}$, depending on fiber composition.

Figure 4 is a plot of the laser beat frequency needed to maximize the Brillouin loss that occurs in the first oven (at $10.1 \mathrm{~km}$, fiber F1) and in the third oven (at $27.4 \mathrm{~km}$, fiber F4) as a function of oven temperature. For these data the rms deviations from the best straight-line fit were 0.93 and $1.12^{\circ} \mathrm{C}$, respectively. From the data, the temperature coefficient of the Brillouin frequency shift was determined to be $1.25 \pm$ $0.10 \mathrm{MHz} /{ }^{\circ} \mathrm{C}$. According to these experimental data, the temperature resolution of this system is $\pm 1^{\circ} \mathrm{C}$.

We have realized a DTS based on Brillouin loss that has a 32.2-km sensing length with a $1^{\circ} \mathrm{C}$ temperature resolution combined with a spatial resolution of $5 \mathrm{~m}$.

The authors gratefully acknowledge the financial support of the UK Science and Engineering Research Council and the Optoelectronics Interdisciplinary Research Centre, Southampton, UK.

\section{References}

1. A. H. Hartog, J. Lightwave Technol. LT-1, 498 (1983).

2. J. P. Dakin, D. J. Pratt, G. W. Bibby, and J. N. Ross, Electron. Lett. 21, 569 (1985).

3. A. H. Hartog, A. P. Reach, and M. P. Gold, Electron. Lett. 21, 1061 (1985).

4. T. Shiota and F. Wada, Proc. Soc. Photo-Opt. Instrum. Eng. 1586, 13 (1991).

5. D. Culverhouse, F. Farahi, C. N. Pannell, and D. A. Jackson, Electron. Lett. 25, 914 (1989).

6. T. Kurashima, T. Horiguchi, and M. Tateda, Opt. Lett. 15, 1038 (1990).

7. X. Bao, D. J. Webb, and D. A. Jackson, Opt. Lett. 18, 552 (1993).

8. T. Horiguchi and M. Tateda, J. Lightwave Technol. 7, 1170 (1989). 